\title{
Is it possible to predict electromagnetic resonances in proteins, DNA and RNA?
}

Irena Cosic ${ }^{1,2^{*}}$, Drasko Cosic ${ }^{2}$ and Katarina Lazar ${ }^{2}$

\author{
* Correspondence: \\ irenacosic@me.com \\ ${ }^{1}$ RMIT University, Melbourne 3000, \\ VIC, Australia \\ ${ }^{2}$ AMALNA Consulting, 46 Second St, \\ Black Rock 3193, VIC, Australia
}

\begin{abstract}
Background: It has been shown that there are electromagnetic resonances in biological molecules (proteins, DNA and RNA) in the wide range of frequencies including $\mathrm{THz}, \mathrm{GHz}, \mathrm{MHz}$ and $\mathrm{KHz}$. These resonances could be important for biological function of macromolecules, as well as could be used in development of devices like molecular computers. As experimental measurements of macromolecular resonances are timely and costly there is a need for computational methods that can reliably predict these resonances.

We have previously used the Resonant Recognition Model (RRM) to predict electromagnetic resonances in tubulin and microtubules. Consequently, these predictions were confirmed experimentally.

Methods: The RRM is developed by authors and is based on findings that protein, DNA and RNA electromagnetic resonances are related to the free electron energy distribution along the macromolecule.

Results: Here, we applied the Resonant Recognition Model (RRM) to predict possible electromagnetic resonances in telomerase as an example of protein, telomere as an example of DNA and TERT mRNA as an example of RNA macromolecules.
\end{abstract}

Conclusion: We propose that RRM is a powerful model that can computationally predict protein, DNA and RNA electromagnetic resonances.

Keywords: Molecular resonances; Proteins; DNA; RNA; Resonant recognition model

\section{Background}

Resonances are ever intriguing processes as they enable energy to be transferred selectively and with minimum loss. Resonances in biological macromolecules (proteins, DNA and RNA) are even more compelling, because they relate to macromolecular biological activity as well as have possible usage in technological developments (molecular computers) [1-11]. Recently, it has been shown that tubulin and microtubules have specific electromagnetic resonances that have a frequency range from $\mathrm{THz}$ to $\mathrm{KHz}$ [11]. Although there are similar examples where these resonances are measured experimentally [8-11], there is a need for theoretical models that can reliably predict these resonances in a variety of different macromolecules (proteins, DNA and RNA). Here we present the Resonant Recognition Model (RRM) [12-16] as a good candidate for such predictions. RRM is a revolutionary new approach proposing that macromolecular activity is based on electromagnetic resonances [12-16]. In our previous work we have used the RRM to predict resonances in tubulin and microtubules and shown

(c) 2015 Cosic et al.; licensee Springer on behalf of EPJ. This is an Open Access article distributed under the terms of the Creative Commons Attribution License (http://creativecommons.org/licenses/by/4.0), which permits unrestricted use, distribution, and reproduction in any medium, provided the original work is properly credited. 
that these resonances are in the frequency ranges from $\mathrm{THz}$ to $\mathrm{KHz}$ [17], which has been experimentally confirmed by a recently published paper [11]. Here, we are applying the RRM to predict possible electromagnetic resonances in other proteins (telomerase), DNA (telomere) and RNA (TERT mRNA). We propose that RRM is a powerful universal tool that can predict protein, RNA and DNA electromagnetic resonances, which is relevant for macromolecular biological function as well as applicable to new, innovative technological devices.

\section{Methods}

\section{Resonant recognition model}

The RRM is based on the findings that certain periodicities within the distribution of energy of delocalised electrons along a protein molecule are critical for protein DNA and RNA biological function and/or interaction with their targets. If possible charge transfer through these macromolecules is introduced, then charge moving through macromolecular backbone or through its 3D structure, like helical structure, can produce electromagnetic radiation, absorption and resonance with spectral characteristics corresponding to energy distribution along the protein. The RRM enables for these spectral characteristics to be determined.

All proteins, DNA and RNA can be considered as a linear sequence of their constitutive elements: amino acids or nucleotides. The RRM model interprets this linear information as a numerical series by assigning each amino acid a physical parameter representing the energy of delocalised electrons of each amino acid and then transforming this numerical series into the frequency domain using Fourier Transform. As the distance between amino acid in a polypeptide chain is $3.8 \AA$, it can be postulated that the points in the numerical sequence derived are equidistant. Therefore, for this numerical analysis the distance between points are set at an arbitrary value $d=1$. The maximum frequency in the corresponding numerical spectrum is $F=1 / 2 \mathrm{~d}=0.5$. The total number of points in the sequence influences the resolution of the spectrum. Thus, for $\mathrm{N}$-point sequence the resolution in the spectrum is equal to $1 / \mathrm{N}$. The $n$-th point in the spectral function corresponds to the frequency of $f=n / N$.

The RRM uses cross-spectral function to extract common spectral characteristics for sequences with the same or similar biological function. The presence of a peak frequency in a multiple cross-spectral function implies that all of the analysed sequences within the group have this frequency component in common. The same approach can be used for DNA and RNA sequences. However, for the characteristic frequencies between proteins and DNA/RNA macromolecules to be compared, it is required to make adjustments for the differences in distances between nucleotides (3.4 $\AA$ ) and amino acids (3.8 $\AA$ ). These adjustments are made on nucleotide sequences spectrum so the final result could be compared with frequency calculations made for the proteins.

Our previous research has shown that all protein, DNA and RNA sequences with a common biological function have the common frequency component [12-16], that represents the characteristic feature for the observed function/interaction. This characteristic frequency is related to the protein biological function [12-16].

Furthermore, it was shown that the proteins and their targets, other proteins, DNA or RNA, have the same characteristic frequency in common. Thus, we propose that the RRM frequencies characterise not only a general function, but also a recognition and 
interaction between the particular protein and its target, which is based on resonant recognition [12-16].

Macromolecular interactions can be considered as resonant energy transfer between the interacting molecules. This energy can be transferred through oscillations of a physical field, possibly electromagnetic in nature. Since there is evidence that proteins, DNA and RNA have certain conducting or semi-conducting properties, a charge, moving through the macromolecular backbone and passing different energy stages caused by different amino acid or nucleotide side groups, can produce sufficient conditions for a specific electromagnetic radiation or absorption. The frequency range of this field depends on a charge velocity. The RRM proposes that charge is travelling through macromolecular backbone at the charge velocity estimated at $7.87 \times 10^{5} \mathrm{~m} / \mathrm{s}[12,13]$. For this velocity and the distance between amino acids in a protein molecule, which is $3.8 \AA$, the frequency range obtained for protein interactions was estimated to be in the range of $10^{13} \mathrm{~Hz}$ up to $10^{15} \mathrm{~Hz}$. For nucleotide sequences, this frequency needs to be adjusted, as distance between nucleotides is $3.4 \AA$. The adjustment has been done within nucleotide sequences to match amino acid sequences. Therefore, the estimated range for both amino acid and nucleotide macromolecules includes infra-red, visible and ultra-violet light. These computational predictions were found to be related to biological function of the macromolecules by comparison with a number of experimental measurements [12-19].

However, if we take into account protein and DNA/RNA complex structures, and in particular alpha helices, the charge transfer is also possible through these structures in the form of solitons [20] (Davydov [21, 22], Hayman [23], Sinkala [24]), excitons (Davydov [21, 22], Sinkala [24], Pang [25],Yomosa [26]) and phonons (Pang [25], Yomosa [26], Ichinose [27], Pang [25]). These other forms of charge transfers are at different velocities ranging from $10^{5} \mathrm{~m} / \mathrm{s}$ for solitons and some exciton all the way down to the speed of sound and small fractions of the speed of sound for phonons. Thus, with the same periodicities within proteins sequences, as determined by the RRM, different modalities of charge transfer can produce different resonant frequencies, which are not necessarily related to their protein biological function, but could be related to the protein and DNA/RNA resonances, in general.

In our previous work, we have applied these charge moving modalities to tubulin and microtubule macromolecules and identified a number of possible electromagnetic resonance frequencies in their macromolecule structures. These results have been experimentally confirmed in research by Bandyopadhyay team, by Sahu et al. [11].

\section{Results and discussion}

Here we applied the RRM approach to three different groups of macromolecules: proteins (telomerases), coding nucleotide sequences (TERT mRNA) and DNA regulatory sequences (telomeres), with the aim to predict electromagnetic resonant frequencies for these three different groups of macromolecules.

\section{Proteins}

As an example of protein macromolecules, the following TERT telomerase proteins were analysed using the RRM approach: Q27ID4 - TERT_BOVIN, O14746 TERT_HUMAN (1-230), O14746 - TERT_HUMAN (325-550), O70372 - TERT_MOUSE and Q673L6 - TERT_RAT. The characteristic common RRM frequency for analysed TERT 
telomerase proteins was found to be at frequency of $\mathrm{f}=0.2930 \pm 0.001$, as presented in Fig. 1 . This result is consistent with our previously published common frequency for human telomerase [28].

When RRM frequencies for telomerase were calculated and used in conjunction with different possible charge velocities through protein, the possible resonant frequencies were in the ranges of $\mathrm{THz}, \mathrm{GHz}, \mathrm{MHz}$ and $\mathrm{KHz}$, as presented in Table 1a.

\section{Coding mRNA}

As an example of coding nucleotide sequences, we analysed the following telomerase TERT mRNA coding sequences, Homo sapiens telomerase reverse transcriptase isoforms: JF896280.1 - Delta2, JF896283.1 - Delta2-8, JF896282.1 - Delta4C, JF896285.1 - Delta413, JF896281.1 - Delta3p-12 and JF896286.1 - INTR1. The common numerical characteristic RRM frequency was found to be at frequency of $f=0.3340 \pm 0.002$, as presented in Fig. 2a. This is in accordance with our previous findings, that all coding nucleotide sequences have the same characteristic frequency of $f=0.3300 \pm 0.002$, describing the common function "coding". As three nucleotides code for each amino acid, it is reasonable to expect that characteristic frequency for "coding" function will reflect triplet codes and will represent periodicity of three nucleotides or frequency of $0.33(1 / 3)$.

To be able to calculate the actual electromagnetic resonant frequencies in nucleotide sequence, it is necessary to adjust for the difference in distances between nucleotides and amino acids, as described above. Therefore, the main numerical frequency peak shifted to the frequency of $f=0.3730 \pm 0.002$, as presented in Fig. $2 b$. The analysis of the mRNA sequences have shown two distinct characteristic peaks at frequency of $f=0.3730 \pm 0.002$ and at frequency of $\mathrm{f}=0.4014 \pm 0.002$. Keeping in mind the different modalities of possible charge transfer through the protein molecule these frequencies (periodicities) can produce specific resonances in the ranges of $\mathrm{THz}, \mathrm{GHz}, \mathrm{MHz}$ and $\mathrm{KHz}$, as presented in Table $1 \mathrm{~b}$. It is interesting to note that TERT and TERT mRNA have similar the second most prominent peak frequency within the range from 0.40 to 0.41 . This could be due to TERT telomerase activity which is also coded within TERT mRNA. Such similarity is causing overlaps between TERT and TERT mRNA across the whole range of electromagnetic resonances related to the second most prominent frequency.

\section{Regulatory DNA}

We have analysed the telomeres as an example of DNA sequences. The following homo sapiens telomeric repeat region isolates were analysed: HQ167745.1 - AE,

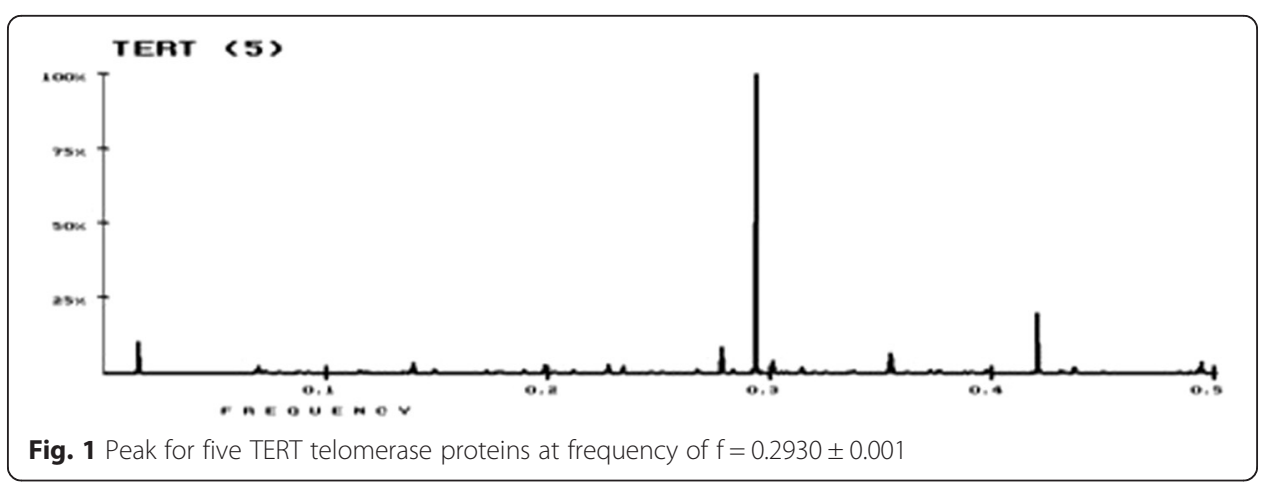


Table 1 Electromagnetic Resonance Frequencies taking into consideration as proposed by different mechanisms of charge velocities for: a. five TERT telomerase proteins; b. six TERT mRNA coding sequences; c. ten telomere sequences, after conversion

\begin{tabular}{|c|c|c|c|c|c|c|c|}
\hline \multirow[t]{3}{*}{ RRM Frequency } & EMf THz per & EMf THz per & EMf GHz per & EMf GHz per & EMf MHz per & EMf MHz per & EMf KHz per \\
\hline & $v=787000 \mathrm{~m} / \mathrm{s}$ & $v=180000 \mathrm{~m} / \mathrm{s}$ & $v=170 \mathrm{~m} / \mathrm{s}$ & $v=68 \mathrm{~m} / \mathrm{s}$ & $v=3.2 \mathrm{~m} / \mathrm{s}$ & $\mathrm{v}=0.34 \mathrm{~m} / \mathrm{s}$ & $v=0.0005 \mathrm{~m} / \mathrm{s}$ \\
\hline & (Cosic) & (Yomosa) & (Davydov) & (Pang) & (Yomosa) & (Ichinose) & (Ichinose) \\
\hline \multicolumn{8}{|l|}{ a. TERT (5) } \\
\hline 0.2930 & 299-308 & $46-47$ & $65-67$ & $26-27$ & $1215-1252$ & 129-133 & 190-196 \\
\hline 0.4194 & $420-429$ & $66-67$ & $92-95$ & $37-38$ & $1747-1785$ & $186-190$ & $272-279$ \\
\hline 0.0161 & $12-21$ & $2-2$ & $2-5$ & $1-2$ & $49-86$ & $5-9$ & $8-14$ \\
\hline 0.2778 & 383-392 & $43-46$ & $61-63$ & $34-35$ & $1151-1180$ & $122-126$ & 180-186 \\
\hline 0.2525 & $261-271$ & $55-57$ & $70-80$ & $21-22$ & $1470-1507$ & $156-160$ & $220-225$ \\
\hline \multicolumn{8}{|c|}{ b. TERT mRNA (6) conversion } \\
\hline 0.3730 & $881-891$ & $58-60$ & $82-85$ & $33-34$ & $1550-1592$ & $165-169$ & $242-249$ \\
\hline 0.4014 & $411-431$ & $63-64$ & $89-91$ & $35-36$ & 1669-1711 & $177-182$ & $261-267$ \\
\hline 0.4902 & 503-513 & $77-78$ & 109-111 & $43-44$ & 2043-2085 & $217-222$ & $319-326$ \\
\hline \multicolumn{8}{|l|}{ c. TELOMERE (10) } \\
\hline 0.1875 & $181-207$ & $28-32$ & $39-45$ & $16-18$ & $737-842$ & 78-89 & $115-132$ \\
\hline 0.1904 & $184-210$ & $28-32$ & $40-45$ & $16-18$ & 749-854 & 80-91 & $117-134$ \\
\hline 0.1768 & $170-196$ & $26-30$ & $37-42$ & $15-17$ & $692-797$ & $78-85$ & $108-125$ \\
\hline 0.1846 & $178-204$ & $27-31$ & $38-44$ & $15-18$ & $785-830$ & $77-86$ & $113-130$ \\
\hline
\end{tabular}




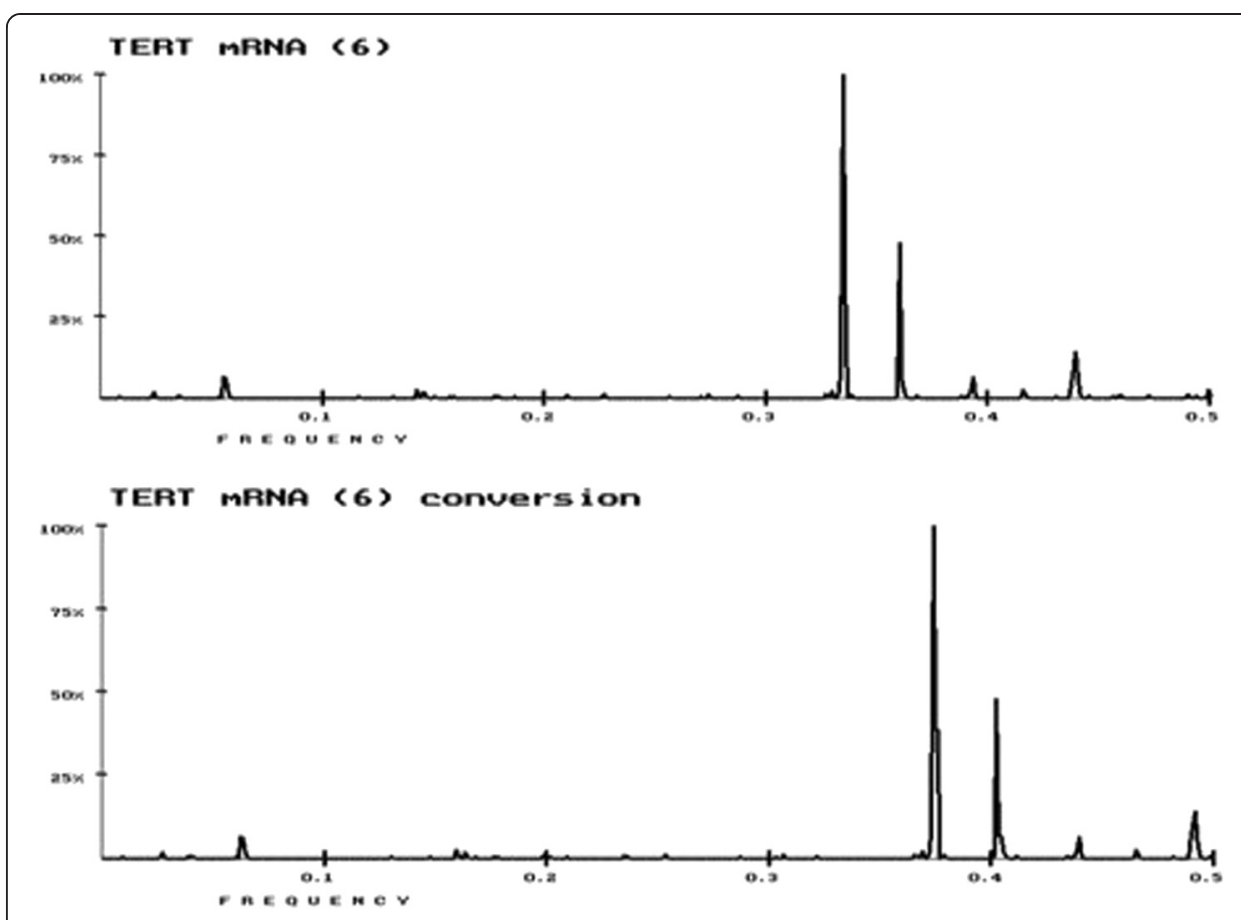

Fig. 2 a Peak for six TERT mRNA coding sequences at frequency of $f=0.3340 \pm 0.002$. b. Peak for six TERT mRNA coding sequences, after conversion, at frequency of $f=0.3730 \pm 0.002$

HQ167740.1 - DVW, HQ167744.1 - KA, HQ167746.1 - KM, HQ167741.1 - KP, HQ167742.1 - LH, HQ167748.1 - SA, HQ167747.1 - SF, HQ167743.1 - VDEC and AF020783.1 - chromosome 20. The main numerical characteristic RRM frequency was found to be at frequency of $\mathrm{f}=0.1875 \pm 0.002$ [28], as presented in Fig. 3. This frequency has been adjusted for the differences in distances between the amino acids in protein and the nucleotides in DNA. When this adjusted numerical characteristic frequency was used in conjunction with different modalities of charge transfer, the possible electromagnetic resonances have been found to be in the ranges of $\mathrm{THz}$, $\mathrm{GHz}, \mathrm{MHz}$ and $\mathrm{KHz}$, as presented in Table 1c. It is interesting to note that telomere have much lower characteristic frequencies than TERT telomerase and TERT mRNA.

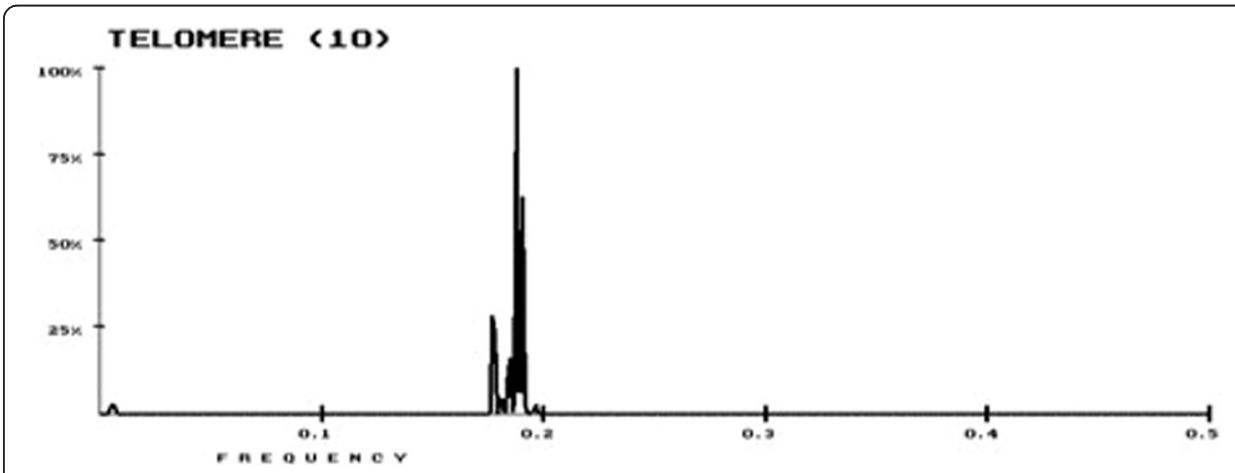

Fig. 3 Peak for ten telomere sequences, after conversion, at frequency of $f=0.1875 \pm 0.002$ 


\section{Conclusion}

We have shown here that the Resonant Recognition Model can be used as universal tool in predicting protein, RNA and DNA electromagnetic resonances in the wide frequency range from $\mathrm{THz}$. $\mathrm{GHz}, \mathrm{MHz}$ and $\mathrm{KHz}$. Keeping in mind that our earlier predictions with tubulin molecules have been experimentally proved [11], we propose that the RRM could be used as a powerful universal method for predicting the electromagnetic resonances in biological macromolecules that could be used in experimental planning and in conjunction with experiments to minimise time and expenditure in exploring such complex macromolecular systems.

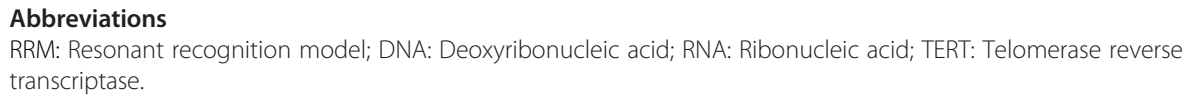

IC developed the RRM model, designed the study, analysed data and wrote most of the paper, DC developed the RRM software, performed calculations and analysed data and $\mathrm{KL}$ was involved in writing the paper and reviewing the text. All authors discussed the results and commented on the manuscript. All authors read and approved the final manuscript.

\section{Acknowledgments}

The authors would like to thank Ms. Alison Kemp for proof reading this paper. The current research work is funded by AMALNA Consulting, registered consulting and research company, Australia.

Received: 14 April 2015 Accepted: 6 May 2015

Published online: 23 May 2015

\section{References}

1. Hameroff S. The brain is both neurocomputer and quantum computer. Cogn Sci. 2007;31:1035-45.

2. Tegmark M. The importance of quantum decoherence in brain processes. Physical Rev E. 2000;61:4194-206.

3. Hameroff S. Ultimate computing-biomolecular consciousness and nanotechnology. North Holland: Elsevier; 1987.

4. Saha AA, Craddock TJA, Tuszynski JA. An investigation of stochastic resonance in tubulin dimers. Biosystems. 2012;107(2):81-7.

5. Sahu S, Ghosh S, Ghosh B, Aswani K, Hirata K, Fujita D, et al. Atomic water channel controlling remarkable properties of a single brain microtubule: Correlating single protein to its supramolecular assembly. Biosens Bioelectron. 2013;47:141-8.

6. Sahu S, Ghosh S, Hirata K, Fujita D, Bandyopadhyay A. Multi-level memory-switching properties of a single brain microtubule. Appl Phys Lett. 2013;102(123701):1-4.

7. Hameroff S, Penrose R. Consciousness in the universe: a review of the 'Orch OR' theory. Physics Life Rev. 2014;11:39-78

8. Craddock TJA, Tuszynski JA, Hameroff S. Cytoskeletal signaling: is memory encoded in microtubule lattices by CaMKII phosphorylation. PLoS Comput Biol. 2012;8(3):1-16. e1002421.

9. Ayoub AT, Craddock TJA, Tuszynski J. Analysis of the strength of interfacial hydrogen bonds between tubulin dimers quantum theory of atoms in molecules. Biophys J. 2014;2:740-50.

10. Dotta BT, Murugan NJ, Karbowski LM, Lafrenie RM, Persinger MA: Shifting wavelength of ultraweak photon emissions from dying melanoma cells: their chemical enhancement and blocking are predicted by Cosic's theory of resonant recognition model for macromolecules. Naturwissenschaften 2014; 101(2) doi:10.1007/s00114-013-1133-3.

11. Sahu S, Ghosh S, Fujita D, Bandyopadhyay A. Live visualizations of single isolated tubulin protein self-assembly via tunneling current: effect of electromagnetic pumping during spontaneous growth of microtubule. Scientific Reports 2014; 4: doi:10.1038/srep07303.

12. Cosic I. Macromolecular bioactivity: is it resonant interaction between macromolecules?-theory and applications. IEEE Trans Biomedical Engineer. 1994;41:1101-14.

13. Cosic I: The Resonant Recognition Model of Macromolecular Bioactivity: Theory and Applications. BirkhauserVerlag 1997.

14. Cosic I. Virtual spectroscopy for Fun and profit. Biotechnology. 1995;13:236-8.

15. Cosic I, Pirogova E:Bioactive Peptide Design using the Resonant Recognition Model. Nonlinear Biomedical Physics 2007; 1(7): doi:10.1186/1753-4631-1-7.

16. Cosic I, Vojisavljevic V, Pavlovic M. The relationship of the resonant recognition model to effects of Low-intensity light on cell growth. Int J Radiat Biol. 1989;56(2):179-91.

17. Cosic I, Lazar K, Cosic D. Prediction of Tubulin resonant frequencies using the Resonant Recognition Model (RRM). IEEE Trans. on NanoBioscience 2014; 12: doi:10.1109/TNB.2014.2365851.

18. Pirogova E, Vojisavljevic V, Istivan T, Coloe P, Cosic I. Review study: influence of electromagnetic radiation on enzyme activity and effects of synthetic peptides on cell trans- formation. MD-Medical Data. 2010;2(4):317-24. 
19. Vojisavljevic V, Pirogova E, Cosic I. The effect of electromagnetic radiation $(550 \mathrm{~nm}-850 \mathrm{~nm})$ on I-lactate dehydrogenase kinetics. Int J Radiat Biol. 2007;83(4):221-30.

20. Ciblis P, Cosic I. The possibility of soliton/exciton transfer in proteins. J Theor Biol. 1997;184:331-8.

21. Davydov AS. Excitons and solitons in molecular systems. Int Rev Cytol. 1987;106:183-225.

22. Davydov AS. Influence of electron-phonon interaction on the motion of an electron in a One-dimensional molecular system. Translated Teoreticheskaya i Matematicheskaya Fizika. 1979;40(3):408-21.

23. Hyman JM, McLaughlin DW, Scott AC: On Davydov's Alpha-Helix Solitons, Long-Time Prediction in Dynamics. John Wiley \& sons 1983, 367-394.

24. Sinkala Z. Soliton/exciton transport in proteins. J Theor Biol. 2006;241:919-27.

25. Pang XF: Theory of Bio-Energy Transport in Protein Molecules and its Experimental Evidences as well as Applications. Higher Education Press and Springer-Verlag 2007.

26. Yomosa S. The exciton in protein. J Phys Soc Jpn. 1963;18(10):1494.

27. Ichinose S. Soliton excitations in alpha-helical protein structures. Chaos, Solitons Fractals. 1991;1(6):501-9.

28. Cosic I, Lazar K, Cosic D. Cellular ageing- telomere, telomerase and progerin analysed using resonant recognation model. MD-Medical Data. 2014;6(3):205-9.

Submit your manuscript to a SpringerOpen ${ }^{\circ}$ journal and benefit from:

- Convenient online submission

- Rigorous peer review

- Immediate publication on acceptance

- Open access: articles freely available online

- High visibility within the field

- Retaining the copyright to your article

Submit your next manuscript at $\boldsymbol{\nabla}$ springeropen.com 